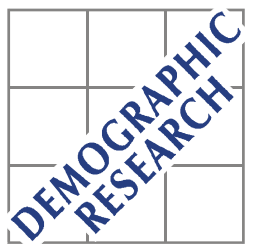

Demographic Research a free, expedited, online journal of peer-reviewed research and commentary in the population sciences published by the Max Planck Institute for Demographic Research Konrad-Zuse Str. 1, D-18057 Rostock · GERMANY www.demographic-research.org

DEMOGRAPHIC RESEARCH

VOLUME 21, ARTICLE 13, PAGES 367-384

PUBLISHED 01 OCTOBER 2009

http://www.demographic-research.org/Volumes/Vol21/13/

DOI: 10.4054/DemRes.2009.21.13

Research Article

Determinants of infant and child

mortality in Zimbabwe:

Results of multivariate hazard analysis

\title{
Joshua Kembo
}

\section{Jeroen K. Van Ginneken}

(C) 2009 Joshua Kembo \& Jeroen K. Van Ginneken.

This open-access work is published under the terms of the Creative Commons Attribution NonCommercial License 2.0 Germany, which permits use, reproduction \& distribution in any medium for non-commercial purposes, provided the original author(s) and source are given credit.

See http:// creativecommons.org/licenses/by-nc/2.0/de/ 


\section{Table of Contents}

$\begin{array}{lll}1 & \text { Introduction } & 368\end{array}$

$2 \quad$ Data and methodology 369

2.1 Model specification 369

$\begin{array}{lll}2.2 & \text { Source of data } & 371\end{array}$

$\begin{array}{lll}2.3 & \text { Statistical methods } & 371\end{array}$

$\begin{array}{llr}3 & \text { Results } & 374\end{array}$

$\begin{array}{lll}3.1 & 374\end{array}$

$\begin{array}{ll}3.2 & \text { Child mortality } \\ \end{array}$

$4 \quad$ Discussion and conclusions $\quad 381$

$5 \quad$ Acknowledgements 382

References 383 


\title{
Determinants of infant and child mortality in Zimbabwe: Results of multivariate hazard analysis
}

\author{
Joshua Kembo ${ }^{1}$ \\ Jeroen K. Van Ginneken ${ }^{2}$
}

\begin{abstract}
This study addresses important issues in infant and child mortality in Zimbabwe. The objective of the paper is to determine the impact of maternal, socioeconomic and sanitation variables on infant and child mortality. Results show that births of order $6+$ with a short preceding interval had the highest risk of infant mortality. The infant mortality risk associated with multiple births was 2.08 times higher relative to singleton births $(p<0.001)$. Socioeconomic variables did not have a distinct impact on infant mortality. Determinants of child mortality were different in relative importance from those of infant mortality. This study supports health policy initiatives to stimulate use of family planning methods to increase birth spacing. These and other results are expected to assist policy makers and programme managers in the child health sector to formulate appropriate strategies to improve the situation of children under 5 in Zimbabwe.
\end{abstract}

\footnotetext{
${ }^{1}$ Joshua Kembo is a Senior Researcher in the Bureau of Market Research (BMR) at the University of South Africa (UNISA). He recently completed his PhD (Epidemiology) in the School of Health Systems and Public Health (SHSPH) at the University of Pretoria. Corresponding contact e-mail address is kemboj@unisa.ac.za.

${ }^{2}$ Jeroen Van Ginneken is Visiting Professor, School of Health Systems and Public Health, University of Pretoria and Honorary Fellow, NIDI, The Hague.
} 


\section{Introduction}

Using the multivariate Proportional Hazards Regression Models this paper presents an analysis of the impact of maternal, socioeconomic and sanitation variables on infant and child mortality. The results presented in this paper were obtained from highly reliable data collected as part of the Demographic and Health Survey conducted in Zimbabwe in 2005-06. The overall purpose of the paper is to determine the relative importance of various maternal, socioeconomic and sanitation variables on infant and child mortality in Zimbabwe between 1996 and 2005. In particular, the study will focus on the relationship between infant and child mortality and birth order, preceding birth interval and, maternal age at birth. Models will then be introduced to control for other relevant socioeconomic and sanitation variables.

Using the results of multivariate analysis of data from 16 countries presented by Hobcraft, McDonald and Rutstein (1984), Cleland and Van Ginneken (1988) demonstrate that shifts in the reproductive pattern (as measured by birth interval, birth order and maternal age) cannot explain the relationship between education and child mortality. However, when Behrman (1988) uses data that permits controlling for the education of a woman's siblings, the education effect nearly disappears. One interpretation of this finding is that the previously reported effects of maternal education may simply be the effect of unobserved familial abilities and motivation passed on from the mother.

Zerai (1996) examined socio-economic and demographic variables in a multi-level framework to determine conditions influencing infant survival in Zimbabwe. He employed Cox regression analysis to the 1988 Zimbabwe DHS data to study socioeconomic determinants of infant mortality. The unique finding was that women's average educational levels in their community exerts a greater influence on infant survival than the mother's educational level. This result supports assertions that child survival is strongly impacted by mass education (Cleland and van Ginneken 1988). However, the author did not show the differential impact of the independent variables on infant and child mortality. The current paper seeks to fill this gap in the existing literature on childhood mortality, by analyzing how child and infant mortality are differently impacted by the aforementioned variables, particularly for Zimbabwe.

Bicego (1990) applied a three-step procedure using proportional hazards regression to estimate trends and determinants of childhood mortality in Haiti. He used the data from the 1987 Mortality, Morbidity and Services Utilization Survey (EMMUS) in Haiti. Maternal education and low age at birth were found to have marked effects on neonatal survivorship but little effect thereafter. Indices that reflect community-level access to child health services were shown to be important especially during childhood. 
Manda (1999) used data from the 1992 DHS in Malawi to study the relationship between infant and child mortality and birth interval, maternal age at birth and, birth order, with and without controlling for other relevant explanatory variables. He also investigated the direct and indirect (through its relationship with birth intervals) effects of breastfeeding on childhood mortality. The study employed proportional hazards models. The results show that birth interval and maternal age effects are largely limited to the period of infancy.

As the child increases in age, the influence of social and economic variables on the mortality risk is enhanced, and the relationship between biodemographic variables and mortality risk is strengthened. The study further shows that breastfeeding status does not significantly alter the effects of preceding birth interval length on mortality risk, but does partially diminish the succeeding birth interval effect.

It is clear from the review of the literature above that the Cox proportional hazards model was rarely used in the study of the determinants of infant and child mortality in Zimbabwe. It is against this background that this paper will show the impact of several independent variables on infant and child mortality. Our results offer an in-depth use of DHS data and are expected to improve the understanding of the mortality situation of children under five in Zimbabwe and of other African countries as well. Our results should be of interest to people working with Zimbabwe and on other studies that analyse child mortality risks. The next section deals with the methodological approach that we used in this study.

\section{Data and methodology}

\subsection{Model specification}

Childhood mortality is analysed in two age periods: mortality from birth to the age of 12 months, which will be referred to as "infant mortality"; and mortality from the age of 12 months to the age of 60 months, which will be referred to as "child mortality". In both cases the dependent variable is risk of death occurring in an age interval in a period, such as from birth to age one, in a calendar year. Based on the Mosley and Chen (1984) determinants of childhood morbidity and mortality framework, the independent variables that we studied in this paper were:

- $\quad$ Maternal (and related) factors: (child's birth order, preceding birth interval, maternal age, child's sex, type of birth),

- $\quad$ Socioeconomic variables: (maternal education, paternal education, wealth index and area of residence), 
- $\quad$ Sanitation: (source of drinking water and toilet facility).

The outcome variable is the hazard ratio (relative risk) of dying in a specific age range of childhood. The age ranges that we use in this paper are:

- Infant mortality $\left({ }_{1} \mathrm{q}_{0}\right)$ : the probability of dying between birth and the first birthday), and,

- $\quad$ Child mortality $\left({ }_{4} \mathrm{q}_{1}\right)$ : the probability of dying between the exact age one and the fifth birthday.

We now discuss the justification for the inclusion of the independent variables in this study. Previous studies have shown that short birth intervals (less than or equal to 18 months), high parity ( 6 or more children), low maternal age (less than 20 years) and high maternal age (35 and more years) adversely impact infant and child mortality (Bicego 1990; Zerai 1996; Manda 1999). We will elaborate more on this point in section 3 when we present the results from the Cox Proportional Hazard Models. Socioeconomic variables such as wealth status determine the availability of nutritional resources, which is especially important because once infants reach the age of 6 months; they can no longer depend on nourishment from breast milk alone.

Mother's education is important because it facilitates her integration into a society impacted by traditional customs, colonialism, and neo-colonialism. Education heightens her ability to make use of government and private health care resources and it may increase the autonomy necessary to advocate for her child in the household and the outside world (Caldwell 1989). Distinct childhood mortality differentials by place of residence (rural-urban) have been observed in Zimbabwe (Zimbabwe Central Statistical Office/ Macro International Inc, 2007). These mortality differences are a result of regional differences in health infrastructure, and communication and disease prevalence conditions. Place of delivery is also an important determinant of mortality, particularly neonatal mortality. Children delivered in modern health facilities usually exhibit lower rates of mortality. However, in some cases, mortality among children delivered in modern facilities is observed to be higher because mothers use these facilities mostly when they have pregnancy complications.

In Zimbabwe, household contamination is still a big problem. Piped water is provided to a minority of households. Only 36 percent of households have water piped into the dwelling, yard or plot, while 5 percent of households use a public tap or standpipe (ibid). Sanitation measures are still not adequate in Zimbabwe. Improvements in hygienic sanitation facilities lower mortality through the mechanism of less exposure of children to contamination making them less susceptible to disease and eventually death. Only 40 percent of households in Zimbabwe have access to improved toilet 
facilities that are not shared with other households (ibid). This evidence confirms the importance of sanitation in the study of determinants of childhood morbidity and mortality.

It is against this background that in this paper we study the selected demographic and socio-economic variables discussed above in order to determine their differential impact on infant and child mortality in Zimbabwe. Other variables from the classical proximate determinants model such as nutrient availability and incidence of injury are not examined because of the absence of sufficient information on the variables themselves from the 2005-06 ZDHS survey data.

\subsection{Source of data}

The study used highly reliable data collected from the 2005-06 ZDHS survey. The 2005-06 ZDHS survey collected data from a sample of 8,907 women aged 15-49 years and 7,175 men aged 15-54 years (ibid). This ZDHS is the fourth comprehensive survey conducted in Zimbabwe as part of the Demographic and Health Surveys (DHS) programme. The DHS are a rich source of data on developing countries in general, and Africa in particular. The empirical analysis in this paper for the independent variables is restricted to 10 years before the 2005-06 ZDHS survey, that is 1996-2005, so that the hazard ratios are based on a sufficient number of cases in each category to ensure statistically reliable estimates.

\subsection{Statistical methods}

The Child Data file that was used in this study was constructed from the Individual Woman's Data file - Individual Recode (IR) from the 2005-06 ZDHS survey using the CASESTOVARS command in SPSS 16.0 (SPSS Inc 2008). The data were adjusted for sampling weights using the WEIGHT command available in SPSS 16.0. The COXREG survival analysis command in SPSS 16.0 was further used to compute the Cox proportional hazard ratios. The significance tests in the hazard models were performed at three levels, that is, " $p<0.05 ", " p<0.01$ " and " $p<0.001$ ".

The Proportional Hazards Model, which stems from the work of Cox (1972), assumes that for an individual with a vector of covariates in $x$, the hazard rate (death rate) at time $t$ is given by:

$$
\mathrm{h}_{\mathrm{i}}\left(\mathrm{t}_{\mathrm{i}} ; \mathrm{x}_{\mathrm{i}}\right)=\mathrm{h}_{\mathrm{o}}\left(\mathrm{t}_{\mathrm{i}}\right) \exp \left(\beta^{\mathrm{i}} \mathrm{x}_{\mathrm{i}}\right)
$$


where $\mathrm{h}_{\mathrm{i}}\left(\mathrm{t}_{\mathrm{i}} ; \mathrm{x}_{\mathrm{i}}\right)$ is the underlying hazard function at time $t$ for $x=0$ (that is, all covariates at their appropriate reference levels) and $\beta^{\mathrm{i}}$ is a vector of unknown coefficients of covariate effects.

Table 1: Absolute and percent distribution of explanatory covariates 1996-2005, (2005-06 ZDHS)

\begin{tabular}{|c|c|c|c|c|}
\hline Maternal covariate & Number of live births & $\%$ & $\begin{array}{c}\text { Number of } \\
\text { under- } 5 \text { deaths }\end{array}$ & $\%$ \\
\hline \multicolumn{5}{|c|}{ Birth order \& preceding } \\
\hline \multicolumn{5}{|c|}{ birth interval } \\
\hline First births & 3,046 & 32.1 & 185 & 30.6 \\
\hline 2-5 and short & 239 & 2.5 & 43 & 7.1 \\
\hline 2-5 and medium & 333 & 3.5 & 28 & 4.6 \\
\hline $2-5$ and long & 4,900 & 51.6 & 271 & 44.9 \\
\hline $6+$ and short & 61 & 0.6 & 15 & 2.4 \\
\hline $6+$ and medium & 74 & 0.8 & 12 & 2.0 \\
\hline $6+$ and long & 836 & 8.8 & 51 & 8.4 \\
\hline \multicolumn{5}{|l|}{ Maternal age } \\
\hline$<20$ years & 2,018 & 21.3 & 131 & 21.7 \\
\hline $20-29$ years & 5,266 & 55.5 & 318 & 52.8 \\
\hline $30-39$ years & 1,953 & 20.6 & 135 & 22.4 \\
\hline $40-49$ years & 254 & 2.7 & 19 & 3.1 \\
\hline \multicolumn{5}{|l|}{ Sex of child } \\
\hline Female & 4,619 & 48.7 & 286 & 47.4 \\
\hline Male & 4,872 & 51.3 & 317 & 52.6 \\
\hline \multicolumn{5}{|l|}{ Type of birth } \\
\hline Multiple & 277 & 2.9 & 51 & 8.5 \\
\hline Singleton & 9,213 & 97.1 & 552 & 91.5 \\
\hline Total & 9,491 & 100.0 & 603 & 100.0 \\
\hline
\end{tabular}


Table 1: (Continued)

\begin{tabular}{|c|c|c|c|c|}
\hline $\begin{array}{l}\text { Socioeconomic } \\
\text { Covariate }\end{array}$ & $\begin{array}{c}\text { Number of live } \\
\text { births }\end{array}$ & $\%$ & $\begin{array}{l}\text { Number of under- } \\
5 \text { deaths }\end{array}$ & $\%$ \\
\hline \multicolumn{5}{|l|}{ Residence } \\
\hline Rural & 6,720 & 70.8 & 440 & 72.9 \\
\hline Urban & 2,770 & 29.2 & 163 & 27.1 \\
\hline \multicolumn{5}{|l|}{ Maternal education } \\
\hline No education & 500 & 5.3 & 29 & 4.8 \\
\hline Primary & 3,689 & 38.9 & 247 & 40.9 \\
\hline Secondary and higher & 5,301 & 55.9 & 328 & 54.3 \\
\hline \multicolumn{5}{|l|}{ Paternal education } \\
\hline No education & 798 & 8.4 & 60 & 10.0 \\
\hline Primary & 2,754 & 29.0 & 168 & 27.9 \\
\hline Secondary and higher & 5,938 & 62.6 & 374 & 62.1 \\
\hline \multicolumn{5}{|l|}{ Wealth status } \\
\hline Poor & 4,292 & 45.2 & 285 & 47.3 \\
\hline Medium & 1,651 & 17.4 & 110 & 18.2 \\
\hline Total & 9,491 & 100.0 & 603 & 100.0 \\
\hline \multicolumn{5}{|l|}{ Piped drinking water } \\
\hline Yes & 3,182 & 33.5 & 194 & 32.1 \\
\hline No & 6,309 & 66.5 & 409 & 67.9 \\
\hline \multicolumn{5}{|l|}{ Flush toilet } \\
\hline Yes & 2,689 & 28.3 & 155 & 25.7 \\
\hline No & 6,801 & 71.7 & 448 & 74.3 \\
\hline Total & 9,491 & 100.0 & 603 & 100.0 \\
\hline
\end{tabular}

The total number of live births between 1996-2005 was 9,491. The number of under-5 deaths during the same period was 603 . Of these deaths, 465 occurred during infancy (0-11 months) and 138 during childhood (12-59 months). The distribution of some explanatory variables over the total sample at risk in the overall age interval 0-59 months is presented in Table 1.

Having discussed the methodological approach in this section, we now present the results in the next section. 


\section{Results}

\subsection{Infant mortality}

The models consisting of all maternal, socioeconomic and sanitation variables are presented in Table 2. Model I consists of maternal reproductive variables only while model II includes socioeconomic variables with maternal reproductive variables. Finally, model III incorporates the remaining sanitation variables.

In model I the mortality risk ratios for birth order and preceding birth interval are in the expected direction. Births of order six or higher with short preceding birth intervals have the highest mortality risk. Infants with these characteristics are 2.75 times more likely to die in infancy relative to births of order two through five with long preceding birth intervals $(\mathrm{p}<0.001)$. Infants of order two through five with short preceding birth intervals experience 37 percent higher risk than infants of order two through five with long preceding birth intervals (although this result did not reach statistical significance). These results, considered together, highlight the importance of parity and birth spacing in determining infant survival. High parity (birth order of $6+$ ) and short preceding birth intervals (intervals less than or equal to 18 months) predispose children to a higher risk of mortality during infancy. Results in model I further show that maternal age of less than 20 years of age increases the risk of infant mortality by 15 percent relative to maternal age between 30 and 39 (not statistically significant). Low (less than 20 years) and high (40-49 years) maternal ages predispose children to elevated mortality risks during infancy. Multiple births are associated with an elevated mortality risk. The infant mortality risk associated with multiple births is 2.08 times greater than among single births $(\mathrm{p}<0.001)$.

Model II extends model I through the addition of socioeconomic controls including place of residence, maternal education, paternal education and wealth index (see Table 2). Infants of order 6 or higher with short preceding interval continue to exhibit the highest risk of death. The probability of such infants dying in infancy is 2.89 times more relative to infants of orders two through five with long preceding intervals. The $U$-shaped relationship of maternal age and infant mortality is not altered in the presence of maternal and socioeconomic variables. Model II presented in Table 2 also shows the impact of socioeconomic variables after controlling for maternal reproductive variables. We observe that socioeconomic variables do not have a distinct impact on infant mortality.

Model III adds controls for two household amenities, namely the piped drinking water and improved toilet facilities. In the presence of maternal and socioeconomic variables the odds of dying for infants born to mothers in households with access to piped drinking water are reduced by 12 percent relative to infants born to mothers in 
households without access to piped drinking water. In the presence of maternal and socio-economic variables, infants born to mothers in households with access to improved toilet facilities are associated with a 38 percent lower risk of dying in infancy compared to those born to mothers in households without access to a such facilities. We further observe that although the odds ratios for piped drinking water and flush toilet are in the expected direction they are both not statistically significant.

\subsection{Child mortality}

The results of the impact of all independent variables (maternal, socioeconomic and sanitation) on child mortality are presented in Table 3 . It is immediately clear that determinants of child mortality are different in relative importance from those of infant mortality. The results for maternal variables in model II of Table 3 are similar to those of model I in the same table.

Table 2: Impact of independent variables on infant mortality, Hazard model estimates of relative risks (RR), 1996-2005 (2005-05 ZDHS)

\begin{tabular}{|c|c|c|c|c|c|c|}
\hline \multirow{3}{*}{ Covariate } & \multicolumn{2}{|c|}{ Model I } & \multicolumn{2}{|c|}{ Model II } & \multicolumn{2}{|c|}{ Model III } \\
\hline & \multirow[b]{2}{*}{ Relative risk } & \multicolumn{2}{|l|}{ Confidence } & Confidence & \multicolumn{2}{|r|}{ Confidence } \\
\hline & & interval & Relative risk & interval & Relative risk & interval \\
\hline \multirow{3}{*}{\multicolumn{7}{|c|}{$\begin{array}{l}\text { Birth order and } \\
\text { preceding birth } \\
\text { interval }^{a}\end{array}$}} \\
\hline & & & & & & \\
\hline & & & & & & \\
\hline First births & 1.101 & $0.859-1.411$ & 1.098 & $0.852-1.416$ & 1.098 & $0.851-1.416$ \\
\hline 2-5 and short & 1.369 & $0.955-1.962$ & 1.387 & $0.958-2.007$ & 1.398 & $0.966-2.025$ \\
\hline 2-5 and medium & 1.474 & $0.950-2.286$ & 1.481 & $0.950-2.310$ & 1.477 & $0.945-2.308$ \\
\hline 2-5 and long & 1.000 & ---------------- & 1.000 & ---------------- & 1.000 & ---------------- \\
\hline $6+$ and short & $2.747^{\star \star \star}$ & $1.544-4.884$ & $2.887^{* * *}$ & $1.598-5.216$ & $2.915^{\star \star \star}$ & $1.613-5.265$ \\
\hline $6+$ and medium & 1.121 & $0.578-2.172$ & 1.146 & $0.584-2.250$ & 1.149 & $0.585-2.256$ \\
\hline $6+$ and long & 1.316 & $0.898-1.929$ & 1.333 & $0.903-1.968$ & 1.337 & $0.906-1.975$ \\
\hline \multicolumn{7}{|l|}{ Maternal age } \\
\hline$<20$ years & 1.147 & $0.796-1.653$ & 1.150 & $0.788-1.678$ & 1.132 & $0.775-1.653$ \\
\hline $20-29$ years & 1.090 & $0.824-1.443$ & 1.092 & $0.816-1.461$ & 1.090 & $0.815-1.459$ \\
\hline $30-39$ years & 1.000 & ---------------- & 1.000 & ---------------- & 1.000 & --------------- \\
\hline $40-49$ years & 1.032 & $0.618-1.725$ & 1.080 & $0.616-1.892$ & 1.081 & $0.617-1.894$ \\
\hline \multicolumn{7}{|l|}{ Sex of child } \\
\hline Female & 0.992 & $0.829-1.187$ & 0.987 & $0.823-1.185$ & 0.979 & $0.816-1.176$ \\
\hline Male & 1.000 & ---------------- & 1.000 & ---------------- & 1.000 & ---------------- \\
\hline
\end{tabular}


Kembo \& Van Ginneken: Determinants of infant and child mortality in Zimbabwe

Table 2: (Continued)

\begin{tabular}{|c|c|c|c|c|c|c|}
\hline \multirow{3}{*}{ Covariate } & \multicolumn{2}{|c|}{ Model I } & \multicolumn{2}{|c|}{ Model II } & \multicolumn{2}{|c|}{ Model III } \\
\hline & \multirow{3}{*}{ Relative risk } & Confidence & \multicolumn{2}{|r|}{ Confidence } & \multirow[b]{2}{*}{ Relative risk } & \multirow{2}{*}{$\begin{array}{c}\text { Confidence } \\
\text { interval }\end{array}$} \\
\hline & & interval & Relative risk & interval & & \\
\hline \multicolumn{6}{|l|}{ Type of birth } & \\
\hline Multiple & $2.080^{\star \star \star}$ & $1.562-2.768$ & $2.086^{\star \star *}$ & $1.563-2.785$ & $2.060^{\star \star \star}$ & $1.541-2.754$ \\
\hline Singleton & 1.000 & -------------- & 1.000 & ------------- & 1.000 & -------------- \\
\hline \multicolumn{7}{|l|}{ Residence } \\
\hline Rural & & & 1.020 & $0.708-1.470$ & 1.323 & $0.765-2.289$ \\
\hline Urban & & & 1.000 & - & 1.000 & - \\
\hline \multicolumn{7}{|l|}{$\begin{array}{l}\text { Maternal } \\
\text { education }\end{array}$} \\
\hline No education & & & 1.000 & 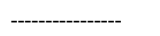 & 1.000 & 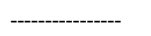 \\
\hline Primary & & & 1.071 & $0.658-1.742$ & 1.074 & $0.660-1.748$ \\
\hline Secondary and & & & 1.039 & $0.619-1.742$ & 1.055 & $0.628-1.770$ \\
\hline \multicolumn{7}{|l|}{ higher } \\
\hline \multirow{2}{*}{\multicolumn{7}{|c|}{$\begin{array}{l}\text { Paternal } \\
\text { education }\end{array}$}} \\
\hline & & & & & & \\
\hline No education & & & 1.000 & 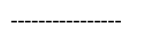 & 1.000 & 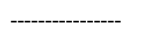 \\
\hline Primary & & & 1.109 & $0.791-1.556$ & 1.116 & $0.796-1.566$ \\
\hline Secondary and & & & 1.117 & $0.800-1.559$ & 1.121 & $0.803-1.565$ \\
\hline \multicolumn{7}{|l|}{ higher } \\
\hline \multicolumn{7}{|l|}{ Wealth status } \\
\hline Poor & & & 1.000 & - & 1.000 & - \\
\hline Middle & & & 1.096 & $0.857-1.402$ & 1.082 & $0.845-1.386$ \\
\hline Rich & & & 1.022 & $0.723-1.444$ & 1.086 & $0.722-1.633$ \\
\hline \multicolumn{7}{|l|}{ Piped drinking } \\
\hline \multicolumn{7}{|l|}{ water } \\
\hline Yes & & & & & 0.885 & $0.597-1.311$ \\
\hline No & & & & & 1.000 & ----------- \\
\hline \multicolumn{7}{|l|}{ Flush toilet } \\
\hline Yes & & & & & 0.629 & $0.348-1.136$ \\
\hline No & & & & & 1.000 & ---------------- \\
\hline
\end{tabular}

a Preceding birth interval: short $<=18$ months, medium 19-23 months, long 24+ months. ${ }^{*} \mathrm{p}<0.05,{ }^{* *} \mathrm{p}<0.01,{ }^{* * *} \mathrm{p}<0.001$

The addition of sanitation variables in model III does not substantially change the impact of maternal and socioeconomic variables observed in model II. The results presented in the full model (model III) demonstrate that first-born children have lower mortality than children of other birth orders. First-born children are 0.57 times less likely to die in childhood relative to children of birth orders two through five with a long preceding birth interval. Furthermore, in the full model, order $6+$ with short 
preceding interval and type of birth do not have any significant effects on child mortality. Significance was observed for both variables in model 2 (see Table 2).

There continues to be an association between residence and child mortality in the presence of maternal and sanitation variables. Living in rural areas increases the risks of childhood mortality by $26 \%$ relative to living in urban areas. This was also found in the infant mortality tables (see Table 2 above). The coefficients depicting the impact of type of residence on infant and child mortality were not statistically significant in either case.

The effect of maternal education, though not significant, implies a decrease in child mortality associated with an increase in maternal schooling. Relative to children whose mothers had no education, mortality among children whose mothers completed primary or secondary education are reduced by 24 percent and 41 percent, respectively. Father's education has a substantial effect on child mortality but not on infant mortality. Completing secondary school reduces the relative risks of child mortality by 33 percent relative to fathers with no formal education.

Table 3: Impact of independent variables on child mortality, hazard model estimates of relative risks (RR), 1996-2005 (2005-06 ZDHS)

\begin{tabular}{|c|c|c|c|c|c|c|}
\hline \multirow{3}{*}{ Covariate } & \multicolumn{2}{|c|}{ Model I } & \multicolumn{2}{|c|}{ Model II } & \multicolumn{2}{|c|}{ Model III } \\
\hline & \multirow[b]{2}{*}{ Relative risk } & Confidence & \multirow{2}{*}{\multicolumn{2}{|c|}{$\begin{array}{cc} & \text { Confidence } \\
\text { interval }\end{array}$}} & \multirow[b]{2}{*}{ Relative risk } & \multirow{2}{*}{$\begin{array}{c}\text { Confidence } \\
\text { interval }\end{array}$} \\
\hline & & interval & & & & \\
\hline \multirow{2}{*}{\multicolumn{7}{|c|}{$\begin{array}{l}\text { Birth order and } \\
\text { preceding birth } \\
\text { interval }^{a}\end{array}$}} \\
\hline & & & & & & \\
\hline First births & $0.549^{*}$ & $0.339-0.890$ & $0.566^{*}$ & $0.345-0.928$ & $0.570^{*}$ & $0.347-0.937$ \\
\hline 2-5 and short & 1.295 & $0.523-3.207$ & 1.317 & $0.530-3.270$ & 1.307 & $0.525-3.252$ \\
\hline 2-5 and medium & 0.816 & $0.329-2.022$ & 0.821 & $0.331-2.038$ & 0.822 & $0.331-2.039$ \\
\hline 2-5 and long & 1.000 & 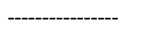 & 1.000 & 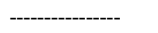 & 1.000 & 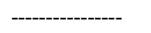 \\
\hline $6+$ and short & 1.071 & $0.146-7.858$ & 0.895 & $0.121-6.624$ & 0.902 & $0.122-6.680$ \\
\hline $6+$ and medium & 0.800 & $0.110-5.830$ & 0.692 & 0.094- 5.093 & 0.719 & $0.098-5.293$ \\
\hline $6+$ and long & 1.177 & $0.619-2.239$ & 0.966 & $0.496-1.881$ & 0.977 & $0.502-1.900$ \\
\hline \multicolumn{7}{|l|}{ Maternal age } \\
\hline$<20$ years & 1.471 & $0.800-2.704$ & 1.461 & $0.788-2.710$ & 1.416 & $0.761-2.636$ \\
\hline $20-29$ years & 0.886 & $0.561-1.398$ & 0.905 & $0.571-1.434$ & 0.889 & $0.560-1.410$ \\
\hline $30-39$ years & 1.000 & 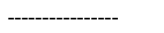 & 1.000 & - & 1.000 & 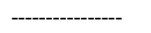 \\
\hline $40-49$ years & 0.294 & $0.069-1.253$ & 0.270 & $0.063-1.158$ & 0.262 & $0.061-1.121$ \\
\hline \multicolumn{7}{|l|}{ Sex of child } \\
\hline Female & 1.011 & $0.732-1.398$ & 1.019 & $0.737-1.409$ & 1.021 & $0.738-1.412$ \\
\hline Male & 1.000 & 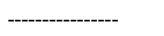 & 1.000 & 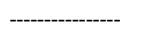 & 1.000 & 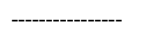 \\
\hline
\end{tabular}


Kembo \& Van Ginneken: Determinants of infant and child mortality in Zimbabwe

Table 3: (Continued)

\begin{tabular}{|c|c|c|c|c|c|c|}
\hline \multirow{3}{*}{ Covariate } & \multicolumn{2}{|c|}{ Model I } & \multicolumn{2}{|c|}{ Model II } & \multicolumn{2}{|c|}{ Model III } \\
\hline & \multirow{3}{*}{ Relative risk } & \multirow{2}{*}{$\begin{array}{c}\text { Confidence } \\
\text { interval }\end{array}$} & \multirow{2}{*}{\multicolumn{2}{|c|}{$\begin{array}{c}\text { Confidence } \\
\text { interval }\end{array}$}} & \multirow[b]{2}{*}{ Relative risk } & \multirow{2}{*}{$\begin{array}{c}\text { Confidence } \\
\text { interval }\end{array}$} \\
\hline & & & & & & \\
\hline \multicolumn{6}{|l|}{ Type of birth } & \\
\hline Multiple & 1.428 & $0.625-3.259$ & 1.510 & $0.660-3.457$ & 1.492 & $0.651-3.419$ \\
\hline Singleton & 1.000 & - & 1.000 & - & 1.000 & - \\
\hline \multicolumn{7}{|l|}{ Residence } \\
\hline Rural & & & 1.135 & $0.598-2.153$ & 1.260 & $0.545-2.912$ \\
\hline Urban & & & 1.000 & - & 1.000 & - \\
\hline \multicolumn{7}{|l|}{$\begin{array}{l}\text { Maternal } \\
\text { education }\end{array}$} \\
\hline No education & & & 1.000 & 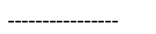 & 1.000 & 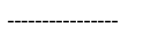 \\
\hline Primary & & & 0.746 & $0.364-1.529$ & 0.764 & $0.373-1.564$ \\
\hline Secondary and & & & 0.567 & $0.264-1.218$ & 0.594 & $0.276-1.276$ \\
\hline \multicolumn{7}{|l|}{ higher } \\
\hline \multicolumn{7}{|l|}{$\begin{array}{l}\text { Paternal } \\
\text { education }\end{array}$} \\
\hline No education & & & 1.000 & - & 1.000 & 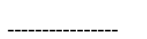 \\
\hline Primary & & & 0.618 & $0.347-1.099$ & 0.614 & $0.345-1.091$ \\
\hline Secondary and & & & 0.675 & $0.390-1.168$ & 0.670 & $0.387-1.159$ \\
\hline \multicolumn{7}{|l|}{ higher } \\
\hline \multicolumn{7}{|l|}{ Wealth status } \\
\hline Poor & & & 1.000 & - & 1.000 & - \\
\hline Middle & & & 1.297 & $0.845-1.989$ & 1.240 & $0.806-1.907$ \\
\hline Rich & & & 1.099 & $0.603-2.001$ & 1.064 & $0.558-2.029$ \\
\hline \multicolumn{7}{|l|}{ Piped drinking } \\
\hline \multicolumn{7}{|l|}{ water } \\
\hline Yes & & & & & 0.606 & $0.330-1.116$ \\
\hline No & & & & & 1.000 & - \\
\hline \multicolumn{7}{|l|}{ Flush toilet } \\
\hline Yes & & & & & $0.401^{* \star}$ & $0.171 \quad 0.940$ \\
\hline No & & & & & 1.000 & - \\
\hline
\end{tabular}

a Preceding birth interval: short <= 18 months, medium 19-23 months, long 24+ months. ${ }^{*} \mathrm{p}<0.05,{ }^{* *} \mathrm{p}<0.01,{ }^{* * *} \mathrm{p}<0.001$ 
Model III further confirms that sanitation variables are more important during childhood than during infancy. Availability of piped drinking water in the dwelling is in the shows a negative impact on child mortality though the alpha is not significant. The odds of dying during childhood for children born in households with access to piped drinking water are reduced by 39 percent relative to those born in households without access to piped drinking water. Availability of improved toilet facilities on child mortality is also in the expected negative direction and is significant. Relative to children born in households with no access to a improved toilets, the relative risks of death for children born in households with access to improved toilets is reduced by $60 \%$ $(\mathrm{p}<0.01)$. This underscores the importance of good quality sanitation in the prevention of diseases such as cholera, diarrhoea and dysentery. Modern sanitation technology ensures the proper disposal of human waste, which is important in preventing the spread of these diseases.

We now compare the results of the impact of birth order, maternal age and maternal education from the 2005-06 ZDHS survey with results from the 1994 and 1999 surveys. These results are presented in Table 4 (bivariate relationships). We do this in order to show the relative change in the impact of these variables on under-five mortality from the period 1985-1994 to the period 1996-2005. The relationship between birth order and under-five mortality is U-shaped indicating higher mortality for both first and higher order births during 1985-1994 and 1990-1999. During 1996-2005 the relationship between birth order and under-five mortality is linear indicating the diminished impact of birth order on under-five mortality. We note that the changes in the relative risks of under-five mortality are not substantial between 1990-1999 and 1996-2005.

The relationships between maternal age and under-five mortality during 19851994 and 1990-1999 are U-shaped. However the U-shaped relationship diminishes and is almost flattened in the 2005-06 survey. For instance, in the 1994 survey the children born to mothers aged less than 20 years experienced 34 percent higher mortality relative to children born to women aged 30-39 years. In the 1999 survey they experienced 21 percent higher mortality, and in the 2005-06 survey the effect of maternal age on underfive mortality is no longer discernible. A similar observation is obtained for the mortality situation of children born to older mothers, that is, those aged 40-49 years. Children born to mothers aged 40-49 years experienced 79 percent higher mortality in the 1994 survey, 61 percent higher mortality in the 1999 survey and 7 percent higher mortality in the 2005-06 survey relative to children born to mothers aged 30-39 years. 
Table 4: Relative risks showing changes in the impact of birth order, maternal age and maternal education on under-five mortality, 1985-1994 (1994 ZDHS), 1990-1999 (1999 ZDHS) and 1996-2005 (2005-06 ZDHS)

\begin{tabular}{lccc}
\hline Variable & $\mathbf{1 9 8 5 - 1 9 9 4}$ & $\mathbf{1 9 9 0 - 1 9 9 9}$ & $\mathbf{1 9 9 6 - 2 0 0 5}$ \\
\hline Birth Order & & & \\
1 & 1.068 & 0.993 & 0.892 \\
$2-3$ & 0.997 & 0.955 & 0.919 \\
$4-6$ & 1.000 & 1.000 & 1.000 \\
$7+$ & 1.084 & 1.223 & 1.081 \\
\hline Maternal age & & & \\
& & & 0.972 \\
$<20$ & 1.343 & 1.207 & 0.944 \\
$20-29$ & 1.214 & 1.122 & 1.000 \\
$30-39$ & 1.000 & 1.000 & 1.069 \\
$40-49$ & 1.794 & 1.605 & \\
\hline Maternal education & & & 1.000 \\
& & & 0.029 \\
No education & 1.000 & 1.000 & 0.790 \\
Primary & 0.845 & 0.434 & \\
Secondary+ & 0.608 & & \\
\hline
\end{tabular}

Authors' calculations are based on data from: Zimbabwe Central Statistical Office/ Macro International Inc, 1995, 2000 and 2007.

Further evidence of the diminishing impact of independent variables on under-five mortality is shown by the changes in the impact of maternal education on under-five mortality from the 1994 survey to the 2005-06 survey. In the 1994 survey, children born to mothers who had completed secondary education experienced 39 percent lower mortality, in the 1999 survey they experienced 57 percent lower mortality and in the 2005-06 survey they experienced 9 percent lower mortality relative to children born to mothers with no formal education.

It could be that these unexpected results are explained by the hypothesis that certain high-risk mothers and subsequently their high-risk births were missing in the 1999 and 2005-06 ZDHS surveys having died due to HIV/AIDS between the time of the 1994 and 2005-06 surveys. It is therefore these "missing mothers" which could explain these observed, unexpected results which show a lack of expected relationships between the independent variables and infant and child mortality in the 2005-06 ZDHS survey and, to a lesser extent, the 1999 ZDHS survey. 
For instance, it could be that there is a group of HIV positive women who were older (30 years and older) and who died and who had children under the age of five years with higher than average mortality. This group could be missing in the 1999 and 2005-06 ZDHS surveys and could not have been interviewed. This means that the observed infant and child mortality rates among women 30 years and older for the period 1990-2005 are actually too low. These rates would have been higher if these missing women could have been interviewed. Further research is required to test the credence of these hypotheses in explaining the observed changes in the determinants of infant and child mortality from 1985-1994 to 1996-2005 in Zimbabwe.

Having presented the results of the multivariate hazard analysis in this section, we now turn to section 4 where we discuss these results and provide concluding remarks and implications of the study.

\section{Discussion and conclusions}

The results of the multivariate analysis presented in this paper show that, in general the strengths of the relationships of the independent (maternal, socioeconomic and sanitation) variables with the dependent variables (infant and child mortality) remain much smaller in the 2005-06 ZDHS survey than in the other ZDHS surveys. For instance, the results from the 1994 and 1999 ZDHS surveys show a larger impact of maternal education on infant mortality than in the 2005-06 survey. The impact of the mother's education on infant mortality completely disappears in 2005-06 in Zimbabwe. These results are rather unexpected and are not in line with observations from other surveys conducted in neighbouring countries.

The multivariate analysis produced only relatively small changes in the strengths of the relationships between independent and dependent variables compared to the bivariate analysis (data not shown in this paper). In the multivariate analysis we found no $U$-shaped relationship between birth order and mortality and maternal age and mortality both in the bivariate and multivariate analysis.

We expect that children born to young mothers (aged less than 20 years) and those born to older mothers (aged 40-49 years) should have higher mortality than those born to mothers aged 20-39 years. The lower risks of child death among children who are first born and those born to mothers aged 40-49 years found in this paper are deviations from the expected mortality pattern and require further investigation. The findings further suggest the following: birth order and preceding birth intervals, maternal age and type of birth are dominant determinants of infant mortality, but they are less pronounced in child mortality. Maternal schooling has a marginal impact on infant mortality. Both maternal and paternal education affects child mortality. 
On sanitation, the findings indicated that the provision of piped drinking water and flush toilets to households has a stronger impact on child mortality than infant mortality. The findings support the thesis that endogenous factors are dominant during infancy while exogenous factors are dominant during the childhood age.

Adult mortality among women of childbearing age more than doubled between 1985-1994 and 1996-1999, and increased by 40 percent between 1996-1999 and 20002005. Close to 60 percent of these extra deaths were to women aged 30-44 years (Zimbabwe Central Statistical Office/ Macro International Inc, 2007). We therefore hypothesize that a group of older women who would have had children with higher than average mortality rates were "missing" from the 1999 and 2005-06 ZDHS surveys. It is probable that there were a number of "missing mothers" from 2005-06 ZDHS survey which not only led to the possible underestimation of the true levels of infant and child mortality, but also to the lack of expected relationships between infant and child mortality and the independent variables in the 2005-06 ZDHS survey. For example, the 1994 and 1999 ZDHS surveys show a stronger impact of maternal education on under-5 mortality. This impact completely disappears in 2005-06. We elaborated on this point in section 3 of this paper.

We conclude that the findings presented in this paper provide further evidence of the importance of practicing birth spacing methods. Women and men living in urban areas or with higher educational levels are more likely to use family planning methods. Thus family and health planning in Zimbabwe should be directed at educating men and women with low educational levels and those in rural areas about the benefits of birth spacing and encouraging them to use birth spacing techniques. In the long run, such policies may be expected run to reduce childhood mortality and possibly socioeconomic variations in mortality, as well. We also saw that in Zimbabwe multiple births are strongly negatively associated with infant survival. This suggests that improving maternal and child health services, screening for high-risk pregnancies and making referral services for high-risk pregnancies more accessible, particularly to the rural women and children, will also contribute to improvement of child survival rates.

\section{Acknowledgements}

This manuscript was drawn from the PhD thesis of the first author. We thank the two anonymous reviewers for their valuable comments that helped to improve this manuscript. Thanks also go to DHS Macro international for their permission to use the 2005-06 ZDHS data set for the analysis in this paper. 


\section{References}

Behrman, J.R. (1988). Nutrition, health, birth order and seasonality: Intrahousehold allocation in rural India. Journal of Development Economics 28(1): 43-62. doi:10.1016/0304-3878(88)90013-2.

Bicego, G. (1990). Trends, age patterns and determinants of childhood mortality in Haiti. [PhD dissertation]. Baltimore: The Johns Hopkins University.

Caldwell, J.C. (1989). Mass education as a determinant of mortality decline. In: Caldwell, J.C. and Santow, G. (eds.). Selected readings in the cultural, social and behavioural determinants of health. Canberra: The Australian National University: 101-109.

Cleland, J.G. and Van Ginneken, J.K. (1988). Maternal education and child survival in developing countries: The search for pathways of influence. Social Science and Medicine 27(12):1357-1368. doi:10.1016/0277-9536(88)90201-8.

Cox, D.R. (1972). Regression models and life-tables. Journal of the Royal Statistical

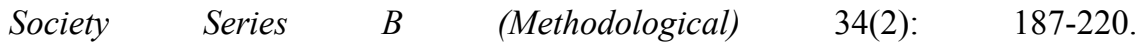
http://www.jstor.org/stable/2985181.

Hobcraft, J.N, McDonald, J.W., and Rutstein, S.O. (1984). Socio-economic factors in infant and child mortality: A cross national comparison. Population Studies 38(2): 193-223. doi:10.2307/2174073.

Manda, S.O.M. (1999). Birth intervals, breastfeeding and determinants of childhood mortality in Malawi. Social Science and Medicine 48(3): 301-312. doi:10.1016/S0277-9536(98)00359-1.

Mosley, W.H. and Chen, L.C. (1984). An analytical framework for the study of child survival in developing countries. Population and Development Review 10:25-45. doi:10.2307/2807954.

SPSS (2008). SPSS, Release 17.0. Advanced Statistical Procedures Companion. Chicago: SPSS Inc.

Zerai, A. (1996). Preventive health strategies and infant survival in Zimbabwe. African Population Studies 11(1): 29-62.

Zimbabwe Central Statistical Office/ Macro International Inc. (1995). Zimbabwe Demographic and Health Survey Country Report. Harare: Central Statistical Office. 
Zimbabwe Central Statistical Office/ Macro International Inc. (2000). Zimbabwe Demographic and Health Survey Country Report. Harare: Central Statistical Office.

Zimbabwe Central Statistical Office/ Macro International Inc. (2007). Zimbabwe Demographic and Health Survey Country Report. Harare: Central Statistical Office. 